\title{
La gestión pública en el Perú en perspectiva histórica (siglos XIX-XXI)
}

\author{
A historical perspective of public management in Peru (19th $-21^{\text {st }}$ centuries)
}

Javier Iván Saravia Salazar

Universidad Nacional Mayor de San Marcos

\section{RESUMEN}

En las siguientes líneas haremos un recorrido por la evolución de lo que actualmente se entiende como gestión pública en nuestro país. Lejos de pretender ofrecer una síntesis sobre el tema, queremos plantear una primera aproximación desde una perspectiva histórica de larga duración. En años recientes, las ciencias sociales han incursionado en el tema con un análisis más profundo de la gestión pública que supera el formalismo jurídico y los tecnicismos administrativos acerca de la modernización del Estado. La gran conflictividad social y los serios cuestionamientos hacia las instituciones públicas y la idoneidad de sus funcionarios, así como los groseros casos de corrupción develados recientemente, ponen en evidencia el serio reto que el Estado peruano tiene de cara al Bicentenario para fortalecer el pacto social con los ciudadanos.

\section{Palabras clave:}

Gestión pública, siglo XIX, siglo XX, siglo XXI, funcionario público, rol del Estado, instituciones políticas.

\section{ABSTRACT}

In the following lines, we will review the evolution of what is currently understood as public management in our country. Far from expecting to offer a summary of the subject, we want to propose a first approach to the subject from a longterm historical perspective. In recent years, social sciences have ventured into the subject with a deeper analysis of public management that goes beyond legal formalism and administrative technical terms of the modernization of the state. The great social conflict, and the serious questioning on public institutions and the suitability of its officials, as well as the recent cases of blatant corruption, revealed the serious challenge of the Peruvian State regarding the forthcoming bicentennial to strengthen the social pact with the citizens.

\section{Keywords:}

Public administration, $19^{\text {th }}$ century, $20^{\text {th }}$ century, $21^{\text {st }}$ century, public official, role of the state, political institutions. 


\section{Introducción}

a modernización del Estado y el fortalecimiento de las instituciones gubernamentales son temas recu-rrentes en la historia de América Latina desde los inicios de su vida política independiente. La adecuada y eficiente administración del Estado ha generado el interés de diversos especialistas, sobre todo de las ciencias del derecho, la administración y la economía, en las cuales existe una perspectiva de análisis básicamente normativo y legalista sobre el desarrollo de lo que hoy se denomina gestión pública.

A la gestión pública se la define como "el conjunto de acciones mediante las cuales las entidades tienden al logro de sus fines, objetivos y metas, los que están enmarcados por las políticas gubernamentales establecidas por el Poder Ejecutivo", y se entiende que "está configurada por los espacios institucionales y los procesos a través de los cuáles el Estado diseña e implementa políticas, suministra bienes y servicios y aplica regulaciones con el objeto de dar curso a sus funciones" (Idea Internacional y Transparencia, 2009, p. 14).

En estas breves líneas, ofreceremos un recorrido histórico sobre cómo ha evolucionado la gestión pública en nuestro país desde la Independencia, con el desarrollo de la estadística y la creación de los primeros ministerios, hasta la actualidad, con los intentos de modernización del Estado.

Si bien la gestión pública es un tema técnico vinculado con el perfeccionamiento de las herramientas de la gerencia estatal y la administración pública, es, en esencia, un tema político. Por ello, en su construcción intervienen diferentes actores y contextos, dentro del marco de la consolidación y la diversificación de los roles del Estado con sus ciudadanos. De un Estado mínimo, con facultades únicamente vinculadas a la policía y la defensa militar, se pasó a un Estado que asumió más roles con los cuales brindar servicios básicos a una masa social cada vez más numerosa. Posteriormente, se convirtió en un Estado planificador, orientado a hacer proyecciones sobre el desarrollo urbano e industrial, y a ser el motor de la economía nacional. Finalmente, el Estado volvió a ser mínimo y se concentró en la focalización de su rol asistencialista a las zonas y poblaciones más vulnerables de la sociedad, y en la regulación de los servicios que brinda el sector privado a la población.
Cada una de estas etapas, y en correspondencia con los roles asumidos por el Estado, demandó la ampliación o reducción del aparato estatal (creación, fusión o eliminación de ministerios y entidades estatales), y, también, cambios en la calidad de los servidores públicos (menos políticos, más técnicos). Estos cambios en el rol del Estado se produjeron, muchas veces, en contra de la voluntad estatal, que, abrumada por las presiones sociales o por los compromisos asumidos ante la comunidad internacional - que le fueron ineludibles-, tuvo que aceptar los nuevos roles exigidos y crear las instituciones para cumplirlos.

\section{La herencia virreinal}

La experiencia peruana después de la Independencia nos presenta la pugna por construir una nación en torno al Estado, y la enorme tara que representó (y representa) la herencia virreinal en la búsqueda de dicho propósito. Según Carl Herbold (1974), el legado administrativo del virreinato en la naciente república peruana tuvo las siguientes características: 1) centralismo; 2) formalismo legal; 3) estatismo; 4) dualismo peruano (existencia del "Perú real" y el "Perú legal"); 5) parasitismo fiscal, y 6) acomodo permanente entre lo tradicional y lo nuevo.

Estas características dificultaron el tránsito hacia un estado moderno - el ideal republicano-y retrasaron la constitución de la profesionalización del servicio público, la descentralización de los servicios públicos y la optimización de la recaudación de impuestos. Además, la lenta implementación de las ideas liberales no significó una ruptura con el pasado ni una apuesta por la modernización del país. Como señala Fernando de Trazegnies (1979), en el siglo XIX se produce una "modernización tradicionalista", que consiste en la: "recepción de un cierto número de elementos capitalistas, ligada a una profunda resistencia a todo cambio en la estratificación social"; es decir, "se quiere devenir capitalista, es decir moderno, pero al mismo tiempo se quiere conservar las jerarquías tradicionales" (p. 30). El aspecto formal del Estado (legal y administrativo) era nominalmente moderno, pero carecía de las características propias de la modernidad, y se convirtió, así, en una prolongación del sistema virreinal basado en privilegios, prácticas clientelares y una visión patrimonialista del Estado. 


\section{El Estado caudillista (1821-1896)}

La vida independiente del país estuvo dominada por intereses particulares y regionalistas que dificultaron la consolidación de un Estado liberal fuerte y administrativamente eficiente. Para Javier Tantaleán (2004), “el Estado de jure era asumido como liberal" y "el Estado de facto interactúa con una infraestructura económica de un nivel mínimo de industrialización y con poderosos intereses exportadores, que fueron variando en el tiempo: guano, salitre, azúcar, algodón, plata y cobre" (pp. 172-173). De esta manera, para Tantaleán, la principal tarea de este Estado "era construirse a sí mismo", pues necesitaba dotarse de marcos jurídicos e institucionales básicos (entes administrativos centrales y departamentales, e infraestructura para servicios básicos y para el comercio), y tomar como referencia, para ello, los modelos administrativos de Europa y Norteamérica.

La alianza entre comerciantes y hacendados hizo viable el asentamiento institucional del Estado. Sin estos grupos, el Estado no hubiese podido mantener su capacidad de dominio, ya que aún eran muy incipientes las instituciones y el manejo administrativo eficiente de los recursos estatales. La recaudación tributaria seguía la lógica de la época: "solo cuando al Estado le son insuficientes los ingresos que vienen de los pobres, se recurre a los ricos; de esta manera los ingresos totales del erario son financiados por los más pobres y los más ricos" (Contreras, 2012, p. 47).

La dinámica era, en sí, sencilla: los hacendados, como grupo, eran una extensión del Estado encargada de la recolección, captación y canalización del tributo indígena, y del control y suministro de brazos para la formación de los ejércitos del caudillo. La recaudación efectiva entre 1830 y 1850 osciló entre los 0,8 y 1,3 millones de pesos, lo que representaba para la época porcentajes de $22 \%$ y $24 \%$ de los ingresos del Estado en las décadas de 1830 y 1840 (Contreras, 2012, p. 47). Las contribuciones indígenas y de las castas eran cobradas por la cadena de subprefectos y gobernadores, $y$, podríamos decir, que su recolección era una de sus ocupaciones principales, por no decir la principal. Los comerciantes, finalmente, actuaban como bancos de crédito del fisco.

\subsection{La organización administrativa}

Desde la Independencia hasta, más o menos, las primeras décadas del siglo xx, la gestión pública se concentraba en cuatro ministerios: Hacienda, Gobierno, Guerra y Marina, e Instrucción Pública, Beneficencia y Negocios Eclesiásticos. Los tres primeros fueron constantes en la organización administrativa de este periodo'. Durante el gobierno de Santa Cruz, se creó el Ministerio de Instrucción Pública, Beneficencia y Negocios Eclesiásticos, que, luego del fin de la Confederación Peruano-Boliviana, desapareció. Agustín Gamarra lo volvió a crear en 1839. Pero no duró mucho, porque, en 1842, el Estado volvió a su organización original de tres ministerios (Quiñones, 2014, p. 3).

La idea del Estado que se tenía por aquellos años era la de uno mínimo, preocupado casi exclusivamente por la seguridad policial, el mantenimiento de las fuerzas armadas y los servicios de justicia. La calidad de vida de la población era un aspecto ajeno al Estado. Un concepto clave de esta manera de entender la gestión pública fue el de las finanzas públicas, concepto heredado de la Revolución Francesa, que se define como la ciencia que estudia la formación y administración del patrimonio del Estado para el cumplimiento de los fines que le son propios ${ }^{2}$. El concepto clásico de las finanzas públicas se regía bajo la doctrina liberal, que estipulaba una presencia mínima del Estado, el cual debía garantizar plena libertad económica para el sector privado. Esta doctrina limitaba los fines del Estado y reducía sus medios de acción; es decir, su patrimonio.

Fue la explotación del guano lo que produjo cambios importantes en la administración pública. En 1856, por ley del 17 de noviembre, se dispuso que esta administración estuviera dividida en cinco ministerios y se estableció las responsabilidades de los ministros y también las relaciones y los límites que estos debían tener respecto al presidente, el resto de instituciones

1 Ocasionalmente, el Ejecutivo optó por unificarlos en una sola secretaría, como sucedió en 1824 y en 1834.

2 A lo largo de todo el siglo xVIII, Europa desarrolló nuevos conocimientos e instrumentos vinculados a la administración del Estado y al manejo apropiado de sus recursos, tales como la taxonomía, la ilustración y las teorías sobre la población. Se puede añadir también el enorme peso que cobró la geografía y, con ella, las expediciones científicas emprendidas por el Estado para conocer sus territorios ultramarinos. El ejemplo por excelencia lo ofreció Francia, que intentó controlar su territorio mediante una cuidadosa y regular inspección de sus recursos, fueran estos hombres o riquezas inanimadas. Desde inicios del siglo xvill, los intendentes franceses se desplazaron por sus jurisdicciones enviando datos para la publicación de un imperfecto pero monumental État de la France. 
públicas y la sociedad en general. Como señala Basadre (2005), con esta ley fue posible establecer, por primera vez, un consejo de ministros en el país (p. 24).

Leticia Quiñones (2014) señala que los siguientes gobiernos no efectuaron modificaciones sustanciales a la organización estatal, pero que sí se enfocaron en potenciar determinadas áreas. José Balta y Manuel
Pardo fomentaron las obras públicas. Pardo, además, contribuyó en la formación de nuevos profesionales al crear la Facultad de Ciencia Política y Administrativa en la Universidad de San Marcos y el Reglamento General de Instrucción Pública de 1876, en el cual se incluía la creación de escuelas técnicas. A partir de dicho reglamento, se creó la Escuela de Ingenieros, que es la actual Universidad Nacional de Ingeniería (UNI).

\section{TABLA 1}

MINISTERIOS Y SUS RESPECTIVAS DIRECCIONES HACIA 1895

\begin{tabular}{|c|c|}
\hline Ministerios & Direcciones \\
\hline \multirow[t]{2}{*}{ Ministerio de Relaciones Exteriores } & Sección Diplomática \\
\hline & Sección Consular \\
\hline \multirow[t]{2}{*}{ Ministerio de Guerra y Marina } & Dirección General de Guerra \\
\hline & Ramo Marina \\
\hline \multirow[t]{6}{*}{ Ministerio de Fomento, Gobierno, Policía y Obras Públicas } & Dirección de Gobierno \\
\hline & Dirección de Policía \\
\hline & Dirección de Correos \\
\hline & Dirección de Telégrafos \\
\hline & Dirección de Obras Públicas \\
\hline & Dirección de Estadística \\
\hline \multirow[t]{4}{*}{ Ministerio de Justicia, Culto, Instrucción y Beneficencia } & Sección Culto \\
\hline & Dirección de Instrucción \\
\hline & Consejo Superior de Instrucción Pública \\
\hline & Sección de Beneficencia \\
\hline \multirow[t]{5}{*}{ Ministerio de Hacienda y Comercio } & Dirección de Administración \\
\hline & Dirección de Rentas \\
\hline & Dirección de Contabilidad General y Crédito \\
\hline & Dirección General de Industria \\
\hline & Sección de Aduanas y Estadística General \\
\hline
\end{tabular}

Fuente: El Peruano (I y II semestre de 1895), Índices; y Quiñones (2014).

\subsection{Los cargos públicos}

Tras la Independencia, la Asamblea Legislativa se ocupó de la organización administrativa del país, creó prefectos donde antes había intendentes y erigió, sobre la base de las divisiones coloniales correspondientes, los departamentos, las provincias y los distritos. Al mismo tiempo, aumentó el número de los empleos públicos. Por tal motivo, el 28 de octubre de 1822, la Junta Gubernativa promulgó una ley que ordenaba que todo funcionario público estaba sujeto a juicio de residencia; y el juez que la tomara, a responsabilidad efectiva por acción popular³.

3 En la actualidad, la Constitución de 1993 señala que la "acción popular" procede "por infracción de la Constitución y de la ley, contra los reglamentos, normas administrativas y resoluciones y decretos de carácter general, cualquiera sea la autoridad de la que emanen (art. 200, i. 5). Por aquellos años, regía la Constitución Política de 1920, que en su art. 157 hace mención a las causas que lo producen, pero no lo conceptualiza, al indicar que "producen acción popular contra los Magistrados y Jueces: la prevaricación, el cohecho, la abreviación o suspensión de las formas judiciales, el procedimiento ilegal contra las garantías individuales y la prolongación indebida de los procesos criminales". 
Los que gobernaban las provincias y de cuya conducta reclamasen los habitantes de ellas, debían ser pesquisados conforme a las leyes y quedar sujetos a las mismas sanciones antes mencionadas.

Por aquellos años, la administración pública estaba conformada por dos clases de agentes administrativos: los funcionarios políticos, tales como prefectos, subprefectos, gobernadores y tenientes gobernadores, que compartían la autoridad con el jefe de Estado, pero eran dependientes del mismo; y los empleados públicos, como ministros de Estado, jefes de ministerio, secretarios, oficiales de oficina y amanuenses, los cuales no compartían autoridad (León y León, 1897, p. 77).

No obstante, los empleos públicos no escaparon al carácter patrimonialista de la organización estatal durante el siglo XIX, como señala Eduardo Quintana (1998). Los gobiernos constitucionales, o los que llegaban al poder luego de una revuelta, dictaban normas para colocar a su clientela en la administración pública o para retirar de ella a sus opositores. Las sucesivas constituciones peruanas del siglo XIX utilizaron un criterio tan vago y poco objetivo para la selección de empleados como el de los "talentos y virtudes".

Por ejemplo, el decreto del 28 de octubre de 1824, dictado por Simón Bolívar, consideraba que los empleados civiles de Lima que no habían emigrado a consecuencia de las defecciones de febrero de ese año habían faltado a la confianza del Gobierno por someterse espontáneamente al poder español. Además, expresaba que los empleos públicos eran de la República y ella debía distribuirlos entre los ciudadanos que le fueran fieles a pesar de las circunstancias, motivo por el cual dispuso que aquellos empleados civiles no tenían derecho a conservar sus destinos (Oviedo, 1861, p. 362) ${ }^{4}$.

El general Luis José de Orbegoso, presidente provisorio, dictó el 14 de mayo de 1834 un decreto que premiaba a los ciudadanos que habían apoyado a su gobierno durante la sedición ocurrida el 3 de enero de ese mismo año y que mandaba que:

Todos los ciudadanos que hayan servido con decisión en la lucha que se ha sostenido y sostiene contra los enemigos de las libertades patrias serán colocados en los empleos de libre elección, calificados que sean sus servicios, y las aptitudes que se requieran para las plazas á que aspiren. (Oviedo, 1861, p. 368)

En diciembre de 1838, durante los años de la Confederación Perú-Boliviana, una circular ordenaba que no se considere para la provisión de empleos, cargos y comisiones a quienes no hicieran constar su enrolamiento en la Guardia Nacional, recientemente creada (Oviedo, 1861, p. 373).

El decreto del 14 de febrero de 1839, dictado en contra de los actos gubernativos de Santa Cruz por el mariscal Agustín Gamarra al asumir la Presidencia de la República, expresaba que, con motivo del estado de guerra en que se había encontrado la República, la elección de muchos empleados se había realizado según "las circunstancias y necesidades del momento", en vez de tomar en cuenta sus méritos y aptitudes personales. Además, afirmaba que ello había sucedido en la época de la usurpación extranjera ${ }^{5}$, por lo que dispuso que: "Se declaran interinos todos los empleos civiles y de Hacienda" (Oviedo, 1861, p. 379). Esta disposición facilitaba la separación inmediata del cargo. En sentido similar, el 15 de noviembre de 1838, el mariscal José de la Riva-Agüero, presidente de la República Nor-Peruana, había expedido un decreto por el que destituyó a los magistrados y funcionarios públicos que firmaron el acta del 24 de agosto de ese año, la cual permitió la elección de Gamarra (Oviedo, 1861, pp. 386-387).

Por su parte, Dancuart (1905) nos infoma que se declaró la invalidez de la destitución de los empleados del ramo de Hacienda ordenada por el gobierno surgido de la revolución de 1855:

\begin{abstract}
Por ley de 11 de Abril de 1861 se reintegró a los empleados titulares del Ramo de Hacienda en los destinos que ocupaban, y de los que habían sido destituidos por la revolución triunfante de 1855, mandando se les pagase los goces de cesantía que debían haber percibido durante el tiempo de su separación. Este percibo se declaró extensivo a los cesantes, por resolución de 10 de Mayo del mismo año. (p. 118)
\end{abstract}

Según el Diccionario de la legislación peruana, de Francisco García Calderón (1879), los empleos, de acuerdo a su periodo de duración, se clasificaban de la siguiente manera:

$4 \quad$ Esta actitud puede entenderse al considerar que aún se desarrollaba la guerra de Independencia. Por ello, es comprensible la discriminación hacia los empleados considerados realistas.

5 En referencia a Santa Cruz y a los chilenos. 
a) Perpetuos o en propiedad, que se concedían por todo el tiempo que el empleado se encontrara apto para desempeñarlos, hasta que solicitara su pensión de jubilación; sólo se perdía por una falta en su desempeño, como el abandono de la función. Al fallecer el empleado propietario dejaba a su familia derecho a recibir una pensión de montepío. La mayoría de empleos civiles y militares eran de este tipo. Estos empleos no eran equivalentes a los nombramientos en los que el titular era denominado propietario para diferenciarlo del suplente, costumbre generalizada en el siglo xIx, por ejemplo, para los cargos de elección ciudadana como miembros del congreso o de concejos municipales.

b) Interinos, que reemplazaban al empleado propietario de la plaza, que había dejado de desempeñarla por razones como una enfermedad, licencia, encargo de otra función, etc.; el plazo de este empleo dependía del momento en que el propietario retomara su cargo o se nombrara un nuevo propietario, según fuera el caso.

c) Temporales, que se ocupaban por un plazo determinado, sin que su desempeño otorgara ningún derecho al concluir el período, por ejemplo el de presidente y de miembro del congreso. (García Calderón, 1879, p. 829g)

El Estado caudillista es un aparato burocrático con predominancia de burocracia militar, en el cual el porcentaje más elevado del gasto se va en el pago de remuneraciones. Un testimonio de la época, una carta de Juan Espinoza a Mauricio Rugendas en 1839, da cuenta de esta situación. En ella se lee lo siguiente: "Me ha asegurado un amigo que ha sido ministro de hacienda en el Perú, que las dos terceras partes de los ingresos del erario, se van en pagar pensiones de favor, empleados inútiles, jubilaciones y estafas" (como se citó en Mc Evoy, 1994, p. 222).

Como recuerda Contreras (2012), desde los primeros años posteriores a la Independencia, "el gasto militar constituía más de dos millones de pesos, vale decir, más de la mitad del total de los ingresos estatales, destinándose el resto a los gastos de recaudación fiscal, la atención de la deuda externa y al gasto político" (p. 44), entendido esto último como la manutención de las oficinas del Poder Ejecutivo: Presidencia de la República y los ministerios. En ese sentido, el control que tuvo el Estado en esos años fue un control básicamente político, garantizado por la manutención de la clase militar, que se erigía como organización clave para asegurar la continuidad del orden republicano. Nicolás de Piérola calculó que, para los años 1851 y 1852, el pago de los sueldos y gastos civiles, y de los ministerios de Hacienda y de Guerra y Marina había consumido más del $70 \%$ y $60 \%$ del presupuesto nacional, respectivamente (Basadre, 1961, p. 979).

Después de las deudas de la Independencia, uno de los capítulos más gruesos de la deuda interna tuvo su origen en la ley de reforma militar, del 12 de diciembre de $1829^{6}$. Esta ley, con el objetivo de disminuir la fuerza armada, enumeró las diferentes categorías de jefes y oficiales que quedaron reformadas; es decir, separadas del servicio activo. A ellas se les concedió, previo reconocimiento y calificación de sus servicios, el derecho a ser pagadas —al igual que a los demás acreedores internos-con bienes nacionales.

José de Morales y Ugalde, ministro de Hacienda Pública, señaló, en 1827, que no conocía a ciencia cierta el monto total de la diversificada deuda interna del país:

\begin{abstract}
así lo siento, y no debo ocultarlo al Congreso, afirmándole que aun el ministerio ignora el monto total de su acrecentamiento (deuda interna: J.T.A.) por estar presentando sucesivamente los acreedores los libramientos dictados por una mano $\tan$ franca, como indiferente a nuestros empeños. (Manifestación del Estado, 1827)
\end{abstract}

\section{El desarrollo de la estadística}

En estas condiciones, el desarrollo de los asuntos estatales y su administración fue más una labor intuitiva que técnica, pero, aun así, existía la necesidad de construir un Estado moderno y de sentar las bases de una institucionalidad para el futuro. A pesar del empirismo en el manejo del Ministerio de Hacienda Pública, de su inestabilidad orgánica y de la falta de técnicas de gestión financiera, los funcionarios de aquel ministerio demostraron un conocimiento intuitivo de los problemas financieros del país, lo que le permitió al Estado afianzarse lenta y gradualmente a lo largo del siglo xIx. En este contexto, la gestión financiera se ceñía a los siguientes principios: 1) minimizar los gastos; 2) no interferir con las iniciativas privadas, y 3) mantener el equilibrio del presupuesto fiscal.

6 Cabe mencionar que, en 1834, se creó el ramo de arbitrios, que consistía en un conjunto de rentas afectas a ciertos créditos privilegiados que el gobierno había reconocido en momentos de apuro y que tenían un sistema especial de intereses y amortización. 
Cumplir con estos fines de manera más precisa, a medida que el Estado empezaba a expandirse, fue lo que propició la aparición y el desarrollo de la estadística, que, pese a ello, tuvo que esperar tres décadas desde el rompimiento con el gobierno español para empezar a configurarse ${ }^{7}$. Como señala Carlos Contreras (2012):

[...] después de la guerra se generalizaron las ideas de que el Estado debía desarrollar una relación de tipo contractual [cursivas del autor] con la sociedad, tomando de ella únicamente lo que ésta consentía, y siempre y cuando esa cesión redundara ulteriormente en el beneficio de ella, puesto que el bien común [cursivas del autor] debía ser, a fin de cuentas, el objetivo de los gobiernos. (pp. 153-154)

Así, después de la Independencia, el Estado entendió a la sociedad "como un organismo en el que podía intervenir" para poder moldearla de acuerdo a los intereses políticos de la élite. Se buscaba la transformación de la sociedad, "a partir del cambio en el origen de las rentas" (Contreras, 2012, p. 158). Con miras hacia las luchas caudillistas, se dieron las condiciones para una planificación desde el Gobierno, lo cual se tradujo en el primer presupuesto y una oficina de estadística.

El primer intento por institucionalizar la recopilación de datos confiables fue la Sociedad de Estadística, creada en 1848. Con ella, se buscaba superar los que, hasta esa fecha, eran considerados como datos inexactos e incompletos, acopiados aisladamente y sin bases fijas y seguras. Su instalación, en agosto de ese año, se llevó a cabo en medio de una gran expectativa. En su reglamento provisional, se estipulaba que la función de la Sociedad era organizar el trabajo estadístico sobre las poblaciones, para redactar, anualmente, los cuadros estadísticos, señalar a las sociedades departamentales la importancia de sus investigaciones, dar su parecer sobre las cuestiones en que fuera consultada, publicar y conservar en sus archivos las tablas estadísticas de cada año, y pedir a las autoridades de la República los datos que considerase necesarios. Años después, se crearía la Sección de Estadística, con oficinas auxiliares en las capitales de cada departamento. El resultado, sin embargo, fue pobre, pues, hasta 1854 , solo se habían censado dos provincias.

En 1873, se creó, por decreto del 31 de octubre, la Dirección de Estadística ${ }^{8}$ dentro del Ministerio de Gobierno, Política y Obras Públicas. Esta dirección estuvo dividida en tres secciones: 1) Estadística de la Población; 2) Estadística del Territorio, y 3) Estadística del Estado. Una de sus primeras medidas fue pedir a prefectos de la República que respondieran a un cuestionario acerca de la situación de sus circunscripciones en materia de población y comercio9. Posteriormente, se le encomendó la realización del primer censo nacional de población en 1876, considerado como el primer censo moderno de la República. A diferencia de los censos anteriores, pensados únicamente para fines tributarios, a la información habitual contenida en los censos coloniales, como raza y estado civil, se añadieron preguntas acerca de la ocupación y la competencia lingüística y alfabética. Estas innovaciones, como bien apunta Contreras (2012), avizora las nuevas áreas en que al Estado le interesaba intervenir.

Otro esfuerzo importante por institucionalizar la recolección de datos y emprender censos con carácter regular se dio con la reorganización del Ministerio de Gobierno, el 30 de abril de 1873. El encargado de realizar esta labor fue el estadístico francés George Marchand, y el encargado de editar el censo y publicarlo, Manuel Atanasio Fuentes.

El régimen revolucionario vio la potencialidad de esta práctica cuando lanzó una serie de encuestas sobre el comercio y las manufacturas para organizar el nuevo Estado. A su vez, este esfuerzo fue llevado a un nivel exponencial con el Directorio, que creó una sección de estadística y terminó asumiendo esta labor como parte medular del Estado. Al mismo tiempo, la estadística (como ciencia del saber) comenzó a desarrollarse al margen de las oficinas gubernamentales y adquirió un status propio dentro de la comunidad científica, como lo demuestran los congresos de especialistas que se llevaron a cabo desde mediados del siglo xix. Las

7 Como nos recuerda Ragas (2016), no es que no haya existido un interés estatal previo, en la era borbónica, por la recopilación de este tipo de información. Ya desde 1760 hasta casi un siglo después, el encargado de esta tarea había sido usualmente un personaje destacado, o el cosmógrafo mayor del Virreinato, como Hipólito Unanue o Gregorio Paredes, respectivamente. La labor de muchos de ellos, por no decir de todos, era solitaria y dependía de la buena voluntad de las autoridades locales, las cuales no siempre comprendían y apoyaban su trabajo (p. 19).

8 Es considerada el antecedente institucional del actual Instituto Nacional de Estadística (INEI).

9 Muchas de las respuestas de las autoridades fueron publicadas en el diario oficial El Peruano, entre el 22 de junio y el 30 de noviembre de 1874 (Contreras, 2012, p. 159). 
reuniones destinadas a intercambiar experiencias sobre este campo se multiplicaron rápidamente: entre 1853 y 1878 tuvieron lugar trece congresos internacionales, los cuales estuvieron vinculados $-\mathrm{y}$ no por casualidad- a las exposiciones universales, que tomaron de la estadística la imagen que presentaban de sus respectivos países al exterior. Hacia finales de siglo, cuando el positivismo reinaba como ideología, la estadística ya contaba con un prestigio muy sólido.

\section{La era de la ampliación de roles del Estado (1896-1930)}

Hacia finales del siglo xIx, como señala Herbold (1973), existía un clima intelectual favorable que cambió la perspectiva en torno al rol del Estado en el país y al modo de hacer política de las élites. El proceso de reconstrucción nacional tras la Guerra del Pacífico y el optimismo de la revolución de 1895 demandaban una reestructuración política del Estado. En el campo académico o intelectual, la reapertura de la Universidad de San Marcos después de la ocupación chilena y el triunfo del positivismo posibilitaron la entrada del pensamiento desarrollista y el darwinismo social entre los intelectuales. El impulso económico de esos años, sumado al protagonismo que Estados Unidos empezó a tener en Sudamérica, contribuyeron a la occidentalización de la cultura y de la política ${ }^{10}$. Estos factores fueron los que dieron forma e influenciaron la conformación de una élite política civil y burocrática, que se materializó en Manuel Pardo y el Partido Civil, cuya influencia y poder político se prolongó hasta 1930, año en que fue derrocado Augusto B. Leguía, último representante político de la República Aristocrática.

Este clima intelectual y sus ideales en torno a lo que debía ser el Estado y cuáles debían ser sus objetivos primordiales quedaron bien sintetizados en el discurso de apertura del año académico en la Universidad de San Marcos, dado por un joven profesor de filosofía, Javier Prado, que consideraba que las bases institucionales de la sociedad peruana debían cambiar para proveer: "[...] el gobierno de la libertad, de la libertad política y la libertad privada; justicia, estabilidad y respeto por las instituciones y seguridad social, un Estado fuerte, uno patriótico, educación, nación trabajadora y sana" (Anales universitarios, XXII).

Glicorio Fernández (1895), en un ensayo que fue su tesis de bachiller en Jurisprudencia en la Universidad de San Marcos escribió que: "el Estado es, sobre todo una persona orgánica y moral y está para perfeccionar la vida nacional: ese es el propósito del Estado" (El fin del Estado, Revista Universitaria, XXII). Joaquín Capelo (1915), por su parte, señaló la necesidad de educar al público en la conducta democrática, así como de implementar y mantener "la gran ley de la renovación" (La vitalidad de las Naciones, Revista Universitaria, X).

Desde el plano netamente político, diferentes voces manifestaron la necesaria reestructuración del sistema político. Manuel Pardo, futuro líder del Partido Civil, invocó una reforma política para promover la vida democrática y preparar al país en la era de la integración colectiva con el mundo (Proclamación de Maestro de la Juventud, Revista Universitaria Tomo XII, 2). Mariano Cornejo, como parlamentario, señaló que lo mejor para el gobierno del país era el régimen parlamentario. En 1915, condenó el crecimiento de lo que él denominaba la burocracia cesarista y demandó la urgencia de la aplicación de la ciencia de la organización en el gobierno del país ${ }^{11}$. José Matías Manzanilla (1903) señaló la urgencia de una reforma electoral y legislativa (El poder legislativo del Perú, Anales Universitarios, Tomo XXXI). Alberto Salomón (1912), quien fue miembro de la cartera de ministros de Leguía, abogó por una representación parlamentaria proporcional por lo que se aunaba a los partidarios de una reforma electoral. Oscar Miró Quesada invocó a la nacionalización o endogenización de la legislación nacional (La nacionalización del derecho por la extensión universitaria, Revista Universitaria, Año VII, N²).

\subsection{ElMinisterio de Fomento}

En 1896, por primera vez desde la Independencia, se creó un nuevo ministerio, el de Fomento, al que se asignaron los ramos de Obras Públicas, que antes había

10 Hay que tener presente, como nos lo recuerda Marcial Rubio (2009), que el liberalismo criollo no se asemejaba al europeo, ni al norteamericano, pues no existía en el Perú una conciencia extendida entre las élites políticas de la necesidad de un Estado moderno y sus virtudes. Entre las personas del pueblo, no había calado la conciencia nacional, al no estar la nación propiamente constituida (p. 39).

11 Al respecto, puede consultarse: El gobierno parlamentario, (Anales Universitarios, Tomo XXIV, 1896, pp. 43-59); Conferencia, (Revista Universitaria, Tomo X, 2, 1915), y Las reformas constitucionales (1915). 
estado bajo la cartera del Ministerio del Gobierno y Policía; Industrias, que antes fue parte del Ministerio de Hacienda, y Beneficencia, que antes fue parte del Ministerio de Justicia. El Ministerio de Fomento surgió dentro de una coyuntura de cambio profundo en el seno del Estado, que tenía como deber principal recuperar la estabilidad económica y reconstruir el país. Antes de la creación del Ministerio de Fomento, un medio de prensa local advertió sobre la necesidad de crear un ministerio donde se reagrupen las direcciones de Obras Públicas e Industrias. Esta institución, según aquel medio, permitiría descargar la recargada labor de los ministerios de Gobierno y Hacienda. Al respecto, opinó que:

El Perú ha avanzado muchísimo en industrias y obras públicas y sin embargo, tenemos hoy el mismo número de ministros que cincuenta años atrás en que éstas estaban en embrión, y en que, la minería, tenía un Tribunal especial que se ocupaba de su despacho. (El Economista, 21 de setiembre de 1895)

El 22 de enero de 1896, el gobierno promulgó la Ley de creación del Ministerio de Fomento, que quedó respaldada por la Constitución de 1860, la cual estipulaba que su ministro debía ser peruano de nacimiento y ciudadano en ejercicio, y que formaría parte del Consejo de Ministros $^{12}$. Entre las consideraciones de su creación, se mencionó el propósito de generar "riqueza pública" (República del Perú, Ministerio de Fomento, 1899). El nuevo ministerio tenía treinta empleados, de los cuales once trabajaban en la Dirección de Fomento y debían promover la producción agrícola y minera, y comenzar a producir estadísticas de ello. Con este ministerio, se consolidó la idea de que al Estado le competía la tarea económica fundamental de construir la infraestructura y producir los bienes y servicios que no estaban dentro del alcance de los agentes particulares.

Leticia Quiñones (2014), quien ha estudiado a fondo este ministerio, señala que los objetivos de la entidad se fueron diversificando con el paso del tiempo. Sin embargo, hasta 1930 se pueden resumir en tres políticas de Estado concretas:

1. El desarrollo de la infraestructura, con la construcción de ferrocarriles, caminos, obras de irrigación, puentes, muelles y edificios públicos, así como la supervisión de y el mejoramiento de los servicios públicos de agua, desagüe, electricidad, telegrafía y telefonía.

2. El desarrollo de las actividades económicas, especialmente en la minería, la agricultura, la ganadería, la piscicultura, la avicultura y las industrias alrededor de ellas; con la formación de nuevos técnicos, a través de la inmigración extranjera, con la reforma y la elaboración del leyes y reglamentos. Así como con la difusión de información relativa a la promoción de productos, inversión en actividades productivas, y en la publicación de todo lo concerniente a proyectos de inversión.

3. El desarrollo social, a través del apoyo a la Beneficencia, al establecimiento de un sistema sanitario efectivo en toda la República, la creación de centros de asistencia médica y antivariólica, la elaboración de leyes en defensa del trabajo obrero, la protección de la niñez y el reconocimiento al indígena y sus comunidades.

12 Como quedaba establecido en la ley del 26 de setiembre de 1862. 
TABLA 2

REORGANIZACIÓN DE LOS MINISTERIOS A PARTIR DE LA CREACIÓN DEL MINISTERIO DE FOMENTO

\begin{tabular}{l|l}
\hline \multicolumn{1}{c|}{ MINISTERIOS } & \multicolumn{1}{c}{ DIRECCIONES } \\
\hline Ministerio de Relaciones Exteriores & $\begin{array}{l}\text { Sección Diplomática } \\
\text { Sección Consular }\end{array}$ \\
\hline Ministerio de Gobierno y Policía & $\begin{array}{l}\text { Dirección de Gobierno } \\
\text { Dirección de Policía } \\
\text { Dirección General de Correos y Telégrafos }\end{array}$ \\
\hline Ministerio de Justicia, Culto, Instrucción y Beneficencia & $\begin{array}{l}\text { Sección Culto } \\
\text { Dirección de Instrucción } \\
\text { Consejo Superior de Instrucción Pública }\end{array}$ \\
\hline Ministerio de Hacienda y Comercio de Guerra y Marina & $\begin{array}{l}\text { Dirección de Administración } \\
\text { Dirección de Rentas } \\
\text { Dirección de Contabilidad General y Crédito } \\
\text { Sección de Aduanas y Estadística General }\end{array}$ \\
\hline Ministerio de Fomento & $\begin{array}{l}\text { Dirección General de Guerra } \\
\text { Ramo Marina }\end{array}$ \\
\hline & $\begin{array}{l}\text { Dirección de Fomento } \\
\text { Sección de Industrias y Colonización } \\
\text { Sección de Beneficencia e Higiene } \\
\text { Sección de Minas y yacimientos } \\
\text { Dirección de Obras Públicas e Irrigación }\end{array}$ \\
\hline
\end{tabular}

Fuente: El Peruano (I y II semestre de 1896), Índices; y Quiñones (2014).

\subsection{El crecimiento del aparato estatal durante el Oncenio}

Durante el periodo de Leguía, se produjo un crecimiento acelerado del aparato estatal, cuya financiación fue producto de la ampliación de la deuda externa y no del crecimiento del aparato económico. Algunas entidades eran totalmente ineficientes, como el caso de los gobiernos municipales regionales (Alayza, 1927, p. 23), contemplados en la carta magna de 1920. Este crecimiento centralizado del aparato estatal no significó un fortalecimiento del Estado, ya que la política económica siguió orientada a la concesión de los recursos naturales del país, vías de comunicación y parte de la administración; $y$, sobre todo, fue incapaz de mejorar el nivel de vida de la población (Placencia, 1985, p. 18).
Un destacado constitucionalista argentino de la época, Carlos Sánchez Viamonte ${ }^{13}$, comentó la creación de instituciones durante el Oncenio:

Sería inútil hablar de instituciones políticas del Perú. Allí la ley consiste en la voluntad personal del presidente. Las Cámaras Legislativas desempeñan una función semejante a la del Senado romano bajo el Imperio. Sus debates constituyen una puja de obsecuencias rivales, un certamen de ditirambos al superdinámico señor Leguía y, en lugar de dictar leyes, convierten sus decisiones en rogativas al Poder Ejecutivo, a fin de que se digne a adoptar tal o cual medida. Como prueba, los diarios de Sesiones.

El Poder Judicial no existe como Poder. Obedece las órdenes del Presidente y, desde hace mucho tiempo, desoye toda reclamación contra la tiranía aunque

13 Profesor de Derecho Constitucional y Ciencia Política, y propulsor de la Reforma Universitaria en Argentina en 1918. 
los actos de ésta afecten la propiedad, la libertad, el honor o la vida de los habitantes". (Como se citó en Planas, 1993, p. 167)

\subsection{La legislación laboral}

Como recuerda Paulo Drinot (2016), hacia finales del siglo xIx e inicios del siglo xx, la industrialización fue de la mano con la civilización y el progreso. Las élites políticas y económicas, especialmente la segunda, concluyeron que era necesario proteger a la clase obrera de influencias que consideraban perniciosas, como el anarquismo y el socialismo, que ya empezaban a calar en la aún precaria organización sindical. Por tal motivo, el Estado comenzó a invertir en sectores de la industria, a la par que creaba y dinamizaba entidades públicas para disminuir la tensión social. En este sentido, por ejemplo, la política laboral del Estado peruano de inicios del siglo xx fue impulsada por abogados, académicos y políticos, que veían en la legislación laboral un componente necesario de la modernidad. En opinión de Drinot (2016):

el Estado peruano en los inicios del siglo xx se desarrolló, ante todo, como un Estado obrero. Es decir, como un Estado de y para la clase obrera, constituida por, y constituyente de, un proyecto de gubernamentabilidad que reflejaba la idea de que la clase obrera era un recurso valioso, un agente de progreso, y que el propósito del Estado era protegerla y manejarla. (p. 72)

En 1919, el Estado implementó la Sección del Trabajo del Ministerio de Fomento, ente que consiguió tecnificar la esfera laboral. Al intervenir en la esfera laboral, el Estado no solo protegía a los trabajadores de lo que consideraba como capitalismo rapaz y socialismo impío, sino que también mejoraba la capacidad de la clase obrera para contribuir el advenimiento de la civilización en el Perú. En la lógica y razonamiento de la época, el Estado debía intervenir en el ámbito laboral con la finalidad de proteger a la clase obrera de las influencias políticas, porque, de no hacerlo, esta clase social podía pasar de ser un agente de progreso a ser una amenaza para la sociedad.

\section{La era del Estado planificador (1930-1990)}

Este periodo se refiere a la necesidad que hubo de orientar la política nacional y las instituciones estatales hacia los nuevos roles asumidos por el Estado, con influencia directa del modelo occidental del estado de bienestar. Ello se materializó en la creación de ministerios e instituciones, así como en la reestructuración de algunos de ellos. Inicialmente, la planificación tuvo una connotación negativa, debido a que era un concepto proveniente de los países adscritos al bloque comunista, pero, lentamente, desde la década de 1930, fue tomada en consideración para reorientar la política nacional y evitar, precisamente, la expansión, en el país, de las ideas comunistas y socialistas.

Óscar R. Benavides creó los ministerios de Salud y Educación en 1935, y desplegó un extenso programa en cada una de estas carteras que sintetizaba el interés del Estado por proporcionar servicios básicos a la población y por ampliar su cobertura para ganar réditos políticos. Se creó el Seguro Social del Obrero en la avenida Grau, en Lima, cuyo imponente edificio reflejaba su compromiso con los más pobres.

En las décadas de 1950 y 1960, hubo un auge de la planificación impulsada por gremios profesionales vinculados a la arquitectura, la ingeniería civil y la medicina, que, eventualmente, influyeron en las políticas públicas y dejaron su impronta a través de obras de infraestructura urbana. Manuel A. Odría, durante su gobierno (el Ochenio) continuó con la senda asistencialista de Benavides y materializó su lema electoral, Salud, educación y trabajo, en una gigantesca y perdurable infraestructura. En materia de salud, construyó el Hospital del Empleado en la avenida Salaverry, en Lima, y otros hospitales en provincia, incluido uno de enormes dimensiones en Tarma. En materia educativa, creó el Fondo de Educación Nacional, con el cual inició la construcción de las grandes unidades escolares. La cobertura en salud y educación se amplió a las masas populares. Durante el gobierno de Belaunde, el arquitecto hizo de la planificación urbana y la conexión del país a través de carreteras sus obras más perdurables. El Gobierno Revolucionario de las Fuerzas Armadas, de alguna manera, hizo de la planificación su signo distintivo, que llevó a casi todas las esferas administrativas. Finalmente, tras el catastrófico primer gobierno de Alan García y el crecimiento desmesurado de la burocracia estatal en la década de 1980, el término volvió a su consideración negativa inicial.

En América Latina en general $-\mathrm{y}$ especialmente en el Perú-, la idea de alentar el progreso y la modernización hizo que surgieran nuevas corrientes de opinión en apoyo a la planificación, la misma que, durante la década de mediados del siglo $\mathrm{xx}$, tenía varias acepciones como: planeamiento, planificación y programación. La planificación fue entonces entendida como programación de 
proyectos de inversión, que solían estar a cargo del sector público. Los profesionales vinculados al planeamiento y desarrollo de viviendas, urbanizaciones, puentes, carreteras y represas fueron los primeros interesados en promover la idea de planificación de proyectos urbanos para encarar la emergente problemática de la vivienda, el uso del suelo y el crecimiento de las ciudades.

Estas iniciativas y perspectivas fueron recogidas por el Estado. Muestras de ello son los diferentes dispositivos legales promulgados en 1948, 1952, 1953 y $1958^{14}$ que permitieron una práctica de la planificación en el ámbito público, como señala Gustavo Saberbein (1984), y que se amplió cuando la actividad empresarial se hizo más importante en el sector público. Los esfuerzos modernizantes no comprometieron al conjunto de la economía. Ante el poco entusiasmo del sector privado, fue el Estado el que se encargó de llevarlos a cabo.

En este sentido, se crea en 1955 el Instituto de Planeamiento al interior de la UNI, en la que se reunían ingenieros civiles, arquitectos y urbanistas, primeros promotores de la actividad planificadora. Fernando Belaunde Terry fundó y dirigió la revista El Arquitecto Peruano entre 1937 y 1966. Muchas de las ideas enunciadas en esta revista conformaron la propuesta política y el plan de gobierno que llevó a cabo en su gestión como presidente.

\subsection{El Sistema Nacional de Planificación ${ }^{15}$}

El Sistema Nacional de Planificación nació en el contexto de la suscripción, por parte del Estado peruano, de la Carta de Punta del Este (8 de agosto de 1961), en el marco de las obligaciones asumidas dentro del programa norteamericano denominado Alianza para el Progreso. El plan de trabajo inicial comprendió tres partes: 1) un análisis de la economía peruana durante el decenio 1950-1960; 2) las bases del plan de desarrollo para el periodo 1961-1970, y 3) los programas de inversiones del Gobierno y entidades oficiales para el periodo 1962-1971.

En lo concerniente al primer punto, el análisis daba cuenta de la irregularidad en el ritmo de crecimiento y en la influencia de las tasas de desarrollo. La irregularidad del crecimiento se explicó por la variación de los precios de nuestras exportaciones; en mayor medida, por los factores endógenos. La tasa de desarrollo se consideró insuficiente para mejorar las condiciones de la economía.

Los objetivos planteados fueron: 1) incremento del PBI a una tasa anual del 5,9\% para un periodo de diez años; 2 ) elevación de la productividad del sector agropecuario; 3 ) incremento de la renta per cápita de los pobladores de la sierra; 4) desarrollo de una política de sustitución de importaciones, y 5) estímulo a la industrialización de las exportaciones.

Se fijaron como prioridades para la inversión: 1) Reforma Agraria; 2) colonización e irrigación; 3) transportes y comunicaciones; 4) electrificación; 5) Programa Social de Vivienda; 6) agua y desagüe; 7) educación; 8) salud; 9) alimentación escolar; 10) Programa de Emergencia en Puno; 11) Plan Nacional de Integración de la Población Aborigen, y 12) desarrollo de la industria.

Se establecieron como organismos del Sistema Nacional de Planificación: 1) el Consejo Nacional de Desarrollo Económico y Social; 2) el Instituto Nacional de

14 El 25 de junio de 1948, se expidió un decreto supremo que creó la Comisión de Estudios y Coordinación de Planes de Desarrollo Económico, con la misión de coordinar todos los planes que contemplen la financiación de préstamos con bancos del exterior y estimular el celo de las reparticiones oficiales encargadas de preparar proyectos que hubieran de financiarse por medio del crédito internacional. El Decreto Supremo N 96 del 10 de octubre de 1952 creó la Comisión para Coordinar, Revisar y Aprobar los Planes de Irrigación, Caminos y Colonización, a la cual se le encargó la tarea de examinar los aspectos técnicos y financieros de proyectos cuya financiación debía hacerse con créditos del exterior. La Comisión estaba presidida por el ministro de Hacienda y Comercio, e integrada por los ministros de Fomento y Obras Públicas, y Agricultura, el contralor general de la República y el director del servicio al cual correspondiera el proyecto. Una vez terminada la coordinación, revisión y aprobación correspondiente, la Comisión debía presentar los estudios definitivos de los proyectos a las instituciones de crédito del exterior para gestionar su correspondiente financiación. El Decreto Supremo № 90 del 16 de agosto de 1955 amplió la cobertura de la Comisión y modificó el trámite de gestión del financiamiento externo. La ampliación de la cobertura abarcó a todos los ministerios, corporaciones (empresas públicas), Banca Estatal de Fomento, municipalidades, beneficencia e, inclusive, a las entidades privadas que requirieran aval del Estado para el financiamiento externo. El trámite de gestión se modificó para que sea el Ministerio de Hacienda y Comercio el que gestionara el proyecto y no la Comisión, para lo cual se designó como miembro decisivo al director general de Hacienda. El 28 de agosto de 1958, se creó la Comisión Técnica de Inversiones Públicas, con la finalidad de asesorar a la Comisión, la misma que solo se pronunció después de recibir los estudios por parte de la Comisión Técnica de Inversiones Públicas. Asimismo, se señaló que la Comisión Técnica sería asesorada por la misión residente del Banco Internacional de Reconstrucción y Fomento contratada por el Estado.

15 Para la información concerniente a este acápite nos remitimos a la obra de Gustavo Saberbein (1984), citada en la bibliografía. 
Planificación, y 3) las Oficinas Sectoriales de Planificación ,como las de: a) Agricultura; b) Pesquería; c) Minería; d) Industria; e) Energía; f) Transporte; g) Comunicaciones; h) Educación; i) Salud; j) Vivienda; k) Trabajo, y l) Turismo.

\subsection{El reformismo militar}

El gobierno militar del general Juan Velasco Alvarado inició un amplio proceso de reformas en el país que seguía el contenido del Plan Inca, con el cual asumió el poder de facto y que duró más de once años. Se produjo un incremento de las estructuras administrativas bajo la visión de un estado proveedor de servicios de todo tipo. La burocracia se expandió, pero se caracterizó por la militarización de los puestos claves y la simultánea expansión de la élite tecnocrática y profesional. La militarización de muchos cargos importantes en la administración pública fue justificada por el régimen debido a la necesidad de reemplazar a un personal deshonesto e ineficiente. Al final del período, hasta el $50 \%$ de la alta oficialidad ocupaba algún puesto en el gobierno fuera de su institución. De este modo, durante doce años, los uniformados estuvieron consagrados a dirigir la sociedad y el Estado. Los asuntos de los cuarteles fueron postergados y las instituciones armadas asumieron el control del país, incluso en mayor medida que un partido político vencedor de una contienda electoral.

Antes de 1968, el sector estatal solo representaba el 7 \% del PBI según tipo de propiedad. Como resultado de las nacionalizaciones, este porcentaje se elevó al $21 \%$ en 1975, año en que cayó el gobierno militar de Velasco. Las cooperativas, gracias a las reformas sociales -entre las que destacó la Reforma Agrariaconformaron el $8 \%$ entre 1968 y 1975 . El sector privado empresarial nacional representaba el $43 \%$ en 1950 , pero su presencia se había reducido a un $27 \%$ en 1975 ante el crecimiento del capital estatal y cooperativo. El capital extranjero mantuvo su peso en ese mismo periodo y pasó de un $10 \%$ al $11 \%$. Sufrió expropiaciones, pero también avanzó en grandes proyectos extractivos (petróleo y cobre) y en algunas manufacturas (automotriz y química).

El Estado creció dantescamente con el proceso de estatizaciones. En este periodo, la absorción estatal de empresas privadas claves de la economía dio origen a empresas estatales emblemáticas: en el rubro pesquero, Pescaperú; en transporte, la Empresa Nacional de Ferrocarriles del Perú (Enaferperú); la empresa estatal Aeroperú para los viajes aéreos y la Empresa Nacional de Puertos (Enapuperú); en comunicaciones, se creó la Empresa Nacional de Telecomunicaciones (Entel), y en hidrocarburos, la empresa estatal Petroperú. Ello evidenció su compromiso con una economía mixta en la que el Estado tuviera un fuerte control, sin eliminar de raíz la inversión privada.

Se intentó una tercera vía entre el capitalismo y el comunismo, y se optó por una economía que combinaba lo privado con lo estatal. No hubo, pues, intención de transferir propiedad a los trabajadores y crear formas extendidas de autogestión. Esta visión económica guardaba más semejanza conceptual con el denominado capitalismo de Estado, en el cual el Estado reemplaza a los trabajadores y la propiedad pública se convierte en sinónimo de socialismo. Este sistema estaba en boga en los países llamados de detrás de la cortina de hierro, pero, en ellos, el Partido Comunista era el representante del pueblo en el Estado, mientras que, en el caso peruano, lo fueron las Fuerzas Armadas.

\subsection{Los límites de la planificación: crisis económica ytensión social}

Tras la vuelta a la democracia en $1980-y$ con una nueva carta magna (1979) producto de un consenso político muy amplio-, Fernando Belaunde Terry regresó a la presidencia con la difícil tarea de reactivar la economía peruana con una estrategia de libre mercado en un contexto de proteccionismo internacional, a la par que ejecutó un proceso de desmontaje de las reformas velasquistas, ya iniciado por Morales Bermúdez. Se desmontó el estatismo orgánico y se desreguló parcialmente el mercado. Pese a las reticencias iniciales, se desmontaron rápidamente las cooperativas creadas bajo el gobierno militar. En consecuencia, la política pública de la liberalización de la economía afectó a las clases populares y medias.

La precariedad en la elección de autoridades y altos funcionarios públicos fue una característica de la década de 1980, como recuerda Sinesio López (1997):

\section{[...] los altos mandos de la administración pública no se seleccionaron de acuerdo a criterios profesionales, sino por el criterio de los cargos de confianza, que se expandieron hasta el nivel de las subdirecciones, y el personal administrativo de apoyo creció verti- ginosamente en cada uno de los gobiernos como una forma de pagar favores a sus leales partidarios, familiares y amigos. (p. 282)}

Para agravar la situación, los cuadros técnico-profesionales de alto nivel no tuvieron la suficiente duración como para desarrollar políticas estables de largo aliento, como se da cuenta en el testimonio de Armando Zolezzi: 
Yo fui director excepcionalmente longevo, duré dos años y dos meses, entre 1980 y febrero de 1982, sin embargo, de febrero de 1982 a la fecha (junio de 1986) me han sucedido ocho directores generales de contribuciones. Entonces, si ustedes toman los últimos cuatro años, hemos tenido ocho directores de contribuciones, ni siquiera el tiempo necesario para calentar el asiento, o sea, no ha habido ni hay una posibilidad real de poder desarrollar una política administrativa en serio. (como se cita en López, 1997, p. 282)

En el gobierno de García, se crearon los gobiernos regionales, elegidos por la ciudadanía de cada región. Sin embargo, esto fue más un gesto político oportunista y demagógico que un compromiso real con un proceso de descentralización. Se dio en un momento difícil para el régimen hacia el término de su mandato y no se les otorgó a estas nuevas autoridades las atribuciones ni competencias -ni mucho menos los recursos económicos - necesarias para consolidarse.

\section{La era de la reforma del Estado (1990 en adelante)}

El tercer periodo iniciado en la década de 1990 significó la vuelta a un Estado mínimo y acotado a funciones de prestación de servicios focalizados y a una participación residual en la economía, únicamente como administrador y regulador, sin capacidad de ejercer su rol de empresario por el principio de subsidiariedad, que sería consagrado en la Constitución de 1993. En este contexto, hubo un proceso de descentralización del poder del Estado y la aparición de nuevas autoridades regionales que dinamizaron y complejizaron la gestión pública.
Tras el desastre económico, político y social que dejó el primer gobierno de Alan García, habían quedado desacreditadas las ideas en torno a un Estado intervencionista y motor de la economía. La credibilidad de las instituciones y la preparación profesional del personal quedó en entredicho. Por ello, la reestructuración del Estado quedó a cargo de "tecnócratas" nacionales y extranjeros formados en el Banco Mundial (BM) y en el Fondo Monetario Internacional (FMI). Además, se adoptó la receta del Consenso de Washington ${ }^{16}$. El Estado había crecido desmesuradamente y se mostraba ineficiente para afrontar la crisis, por lo cual era necesario, aparte de modificar el marco normativo, crear instituciones diferentes o reorganizar las ya existentes, así como dotar a los funcionarios públicos de un nuevo tipo de perfil, más acorde al rubro de servicios del sector privado.

Como acertadamente señala Francis Fukuyama (2004), “la política del siglo xx estuvo tremendamente determinada por las controversias acerca del tamaño y la fuerza de Estado. Durante los tres primeros cuartos del siglo xx, el tamaño, las funciones y el alcance del Estado aumentaron desmesuradamente, lo que ocasionó que los aspectos orgánicos de los Estados variaran sustantivamente desarrollándose y creciendo en complejidad" (Fukuyama, 2004). Fue un fenómeno general, al margen de las características de cada país, tanto en su grado de desarrollo como en la orientación ideológica que regía su vida política, en pleno contexto de las guerras mundiales y la posterior Guerra Fría.

En el siguiente cuadro, ofrecemos una clasificación simplificada de las funciones del Estado que se consolidaron en el siglo xx:

16 Las ideas del Consenso de Washington se explicitan en el siguiente decálogo: 1) disciplina fiscal; 2) reorganización de las prioridades del gasto público; 3) reforma tributaria; 4) liberalización de las tasas de interés; 5) tipo de cambio competitivo; 6) liberalización del comercio y apertura del mercado externo; 7) liberalización de las inversiones extranjeras; 8 ) privatizaciones; 9) desregulaciones, y 10) derechos de propiedad. Para los fines de este artículo, nos concentraremos únicamente en los puntos 2,3 y 5 , por ser los más directamente involucrados al tema que abordamos. 
TABLA 3

FUNCIONES DEL ESTADO SEGÚN FRANCIS FUKUYAMA

\begin{tabular}{|c|c|l|}
\hline \multicolumn{2}{|c|}{ FUNCIONES DEL ESTADO } \\
\hline \multicolumn{1}{|c|}{ FUNCIONES MÍNIMAS } & \multicolumn{1}{|c|}{ FUNCIONES INTERMEDIAS } & \multicolumn{1}{|c|}{ FUNCIONES DINÁMICAS } \\
\hline Suministro de bienes públicos puros: & $\begin{array}{c}\text { Abordar los efectos externos: } \\
\text { - Defensa, ley y orden }\end{array}$ & Política industrial \\
- Derechos de propiedad & Educación, medio ambiente & Generar riqueza \\
- Gestión macroeconómica & Corregir la educación imperfecta: & \\
- Salud pública & Seguros, reglamentación & \\
& imperfecta, Seguridad Social & \\
Aumentar la equidad: & & \\
- Protección de los pobres & & \\
\hline
\end{tabular}

Fuentes: Informe sobre el Desarrollo (Banco Mundial, 1997), Fukuyama (2004)

Elaboración propia

Fukuyama (2004) señala que:

Las organizaciones del sector público producen fundamentalmente servicios, y medir la productividad del sector de servicios resulta, dada su naturaleza, extremadamente complejo. El asunto del control y transparencia es ya bastante complicado en las organizaciones del sector privado, donde al menos se dispone de cotas de rentabilidad para calcular el rendimiento, pero resolverlo se presenta como una tarea prácticamente imposible en muchas de las producciones del sector público. Si el rendimiento no puede medirse con precisión, no puede haber, en última instancia, mecanismo formal alguno para garantizar la transparencia y la rendición de cuentas. (p. 88)

\subsection{Las políticas sociales focalizadas y sectoriales}

Hay que distinguir dos momentos: uno que va de la década de 1990 al año 2000, caracterizado por la austeridad en el gasto público; y el otro, que se inicia con la nueva centuria y que implica un aumento paulatino de la inversión pública en sectores clave como educación, salud e infraestructura.
En la década de 1990, se eliminaron los subsidios directos, y el gasto público en educación, salud e infraestructura se redujo drásticamente. Aparecieron, en este contexto, entidades públicas orientadas a reducir el índice de pobreza o, en todo caso, a solucionar algunos problemas básicos de la población en extrema pobreza. Programas tales como: 1) el Programa de Apoyo al Ingreso Temporal (PAIT); 2) el Programa Municipal del Vaso de Leche; 3) el Programa de Salud Alimentaria (Presa); 4) el Programa de Emergencia Social ${ }^{17}$ (PES); 5) el Fondo de Compensación y Desarrollo Social (Foncodes), y 6) el Programa Nacional de Asistencia Alimentaria (Pronaa) ${ }^{18}$, por citar algunos ejemplos. Este tipo de instituciones requerían de profesionales más vinculados a la gerencia para su adecuada administración, y personal orientado a temas muy concretos para aplicar un modelo en base a resultados cuantificables.

Este esquema de administración no ha variado mucho en la actualidad $y$, aunque muchas de sus instituciones han cambiado su denominación o nombre, estas no han salido del enfoque de atención sectorial y orientada a un sector focalizado de la población. Muestra de ello es el programa Juntos, del pasado gobierno de Alejandro Toledo, y los programas Pensión 65 y Beca 18, del gobierno de Ollanta Humala.

17 Tenía como finalidad proteger a la población más vulnerable a través de la ejecución de programas de orden alimentario y de salud.

18 Al reactivarse el Ministerio de la Presidencia, el Foncodes y el Pronaa fueron adscritos a dicho ministerio con el propósito de concentrar la gestión de diversos programas orientados a atender a la población en situación de pobreza. 


\subsection{La renovación de los ministerios}

Nuevos ministerios fueron creados con el cambio de centuria: 1) el Ministerio de la Producción (2002); 2) el Ministerio del Ambiente (2008); 3) el Ministerio de Cultura (2010), y 4) el Ministerio de Desarrollo e Inclusión Social (2011).

Además, muchos ministerios sufrieron cambios interesantes, no solo desde una perspectiva nominal, sino que incorporaron nuevas necesidades del Estado, exigencias sociales y emergentes demandas sociales. Así, en el 2001, el Ministerio de Trabajo y Comunidades cambió su nombre a Ministerio de Trabajo y Promoción del Empleo del Perú. El Ministerio de la Mujer y del Desarrollo Humano (Promudeh), creado en el año 1996, se convirtió en el Ministerio de la Mujer y Desarrollo Social (Mimdes) en el 2002, para, finalmente, en el 2012, convertirse en el Ministerio de la Mujer y Poblaciones Vulnerables (MIMP).

Aparecieron también nuevas entidades dentro de los ministerios, con labores de supervisión específica, como la Autoridad Nacional del Agua (ANA), creada en el año 2008 como organismo constitucional autónomo, adscrito al Ministerio de Agricultura y encargada de realizar las acciones necesarias para el aprovechamiento multisectorial y sostenible de los recursos hídricos por cuencas hidrográficas.

\subsection{El Estado regulador}

La intervención del Estado en la economía disminuyó de forma drástica en este periodo, pues se entendía que el sector privado dirigiría la economía y el Estado asumiría un rol regulador. Por ello, se mantuvieron y fortalecieron instituciones relacionadas a la política macroeconómica y al funcionamiento del Estado; entre ellas, el Banco Central de Reserva del Perú (BCR), la Superintendencia Nacional de Aduanas y Administración Tributaria (Sunat), y el Instituto Nacional de Defensa de la Competencia y de la Protección de la Propiedad Intelectual (Indecopi). Del mismo modo, surgieron nuevas instituciones con este rol regulador, como el Organismo Supervisor de la Inversión en Energía y Minería (Osinergmin), creada en 1996.

Nuevos entes de justicia aparecieron en este periodo, como la Defensoría del Pueblo, en 1993, y el Tribunal
Constitucional, en 1996, máximo intérprete de la Constitución e institución que reemplazó al Tribunal de Garantías Constitucionales ${ }^{19}$.

\subsection{Hacia un servicio público eficiente}

A comienzos de la década de 1990, se desarrolló un proceso de reducción del aparato administrativo, que implicó, según algunas estimaciones, el despido de 450000 trabajadores. En ese sentido, se fomentó la flexibilidad del empleo público y la modernización institucional. Asimismo, se cerró el ingreso a la carrera administrativa, se habilitó la utilización en el ámbito público del régimen laboral privado (Decreto Legislativo 728) y se propagó la modalidad de servicios no personales. En este proceso, fue disuelto el Instituto Nacional de la Administración Pública, que encabezaba el proceso de la reforma de la administración pública.

En el 2008, se creó la Autoridad Nacional del Servicio Civil (SERVIR) como organismo técnico especializado y rector del Sistema Administrativo de Gestión de Recursos Humanos del Estado, encargado de establecer, desarrollar y ejecutar la política del Estado respecto del servicio civil en materia de organización del trabajo y su distribución, gestión del empleo, rendimiento y evaluación, compensación, desarrollo y capacitación, entre otros. Hoy tiene como una de sus principales funciones la elaboración de propuestas normativas del sistema, entre las que se incluye la propuesta de un modelo de servicio civil para la reforma. Es el intento más serio del Estado por tener personal capacitado y elegido con criterios exclusivamente meritocráticos. Sin embargo, ha tenido un recibimiento poco entusiasta por parte de los funcionarios públicos y aún no recibe el apoyo gubernamental ni ciudadano necesario para que pueda implementarse correctamente.

\subsection{Ladescentralización y los gobiernos regionales}

El ideal de descentralización planteado en la década de 1980 se materializó en el nuevo milenio con la bonanza económica que experimentó el país por el alza sostenida del precio de los minerales en el mercado internacional en esta última década. Ello permitió destinar fondos, a través del canon minero, a los diferentes gobiernos

19 Durante el gobierno de Alberto Fujimori algunos miembros del Tribunal Constitucional fueron separados de la institución por no alinearse con los intereses del hoy condenado expresidente. Posteriormente, los magistrados fueron repuestos por mandato de la Corte Interamericana de Derechos Humanos. 
regionales para que se emprendieran obras diversas en beneficio de la región y sus habitantes. Dicho sistema, lejos de contribuir positivamente, evidenció problemas no previstos. Uno de ellos fue la poca capacidad de gestión e insuficiencia de personal técnico en las regiones, personal que fuera capaz de proponer proyectos viables y pertinentes para la realidad y necesidades de la región y sus pobladores. El rostro de la corrupción, a su vez, se hizo más evidente, al hacerse repetitivos los casos de nepotismo, malversación de fondos y tráfico de influencias en las diferentes regiones que se beneficiaron con el canon y que destinaron cuantiosos fondos en obras con sobrecostos, deficiencias, e, inclusive, totalmente innecesarias, cuando no inútiles, para las necesidades de la población.

\section{A modo de conclusión}

En este rápido y apretado resumen, notamos que uno de los principales inconvenientes para la consolidación de una adecuada gestión pública en nuestro país ha sido y es la poca capacidad de gestión y preparación de los funcionarios públicos al momento de asumir los retos que la sociedad y la administración les exige. Las características de nuestra historia, llena de golpes de Estado y quiebres del orden democrático, han impedido el desarrollo de una carrera de servicio público eficiente que pueda ser transversal a las instituciones y legada a través del tiempo. Pese a los esfuerzos, en las últimas décadas, por crear un servicio civil eficiente, las redes clientelares y la corrupción parecen ser más fuertes que los bien intencionados proyectos de reforma.
El rol del Estado, a través del tiempo, se ha reconfigurado constantemente por una serie de factores que provienen de las mismas necesidades del Estado para lograr su adecuado funcionamiento, de las exigencias de la comunidad internacional, de las presiones de los actores políticos (partidos políticos, Fuerzas Armadas, grupos económicos), o de la misma sociedad organizada en asociaciones y sindicatos. La gestión pública debe orientarse en función al rol que el Estado asuma para, dinamizar las instituciones y adaptar al personal a las nuevas necesidades.

Se aprecia que, a través de los años, la presencia de la corrupción en los organismos estatales ha sido criticada y enfrentada, pero, lastimosamente, los cambios institucionales que ha sufrido el Estado en su constante diversificación de roles no ha venido acompañada del fortalecimiento de las instituciones fiscalizadoras, a las que no se les ha brindado las atribuciones, ni la autonomía ni la continuidad suficientes para hacerle mella. Los recientes acontecimientos en nuestro país muestran vivamente como la incompetencia y la corrupción pueden causar más destrucción que los fenómenos naturales.

Es en esta línea de ideas que debemos replantear una vez más el rol del Estado, en un contexto de insatisfacción ciudadana frente a un modelo de gestión pública, bien intencionado sí, pero sin instituciones fiscalizadoras adecuadas y sin personal idóneo para el servicio público. Este modelo se convierte, así, en un escollo difícil de superar para conseguir la tan anhelada reforma y la modernización del Estado. Es esta una más de nuestras tareas pendientes de cara al bicentenario.

\section{Referencias}

Alayza, T. (1927). Derecho Constitucional General y del Perú. Lima: Editorial Villarán.

CAPElo, J. (1915). La vitalidad de las Naciones. Revista Universitaria (10).

Constitución para la República del Perú. (1920). Recuperado de http://www.leyes.congreso. gob.pe/

Constitución Política del Perú. (1993). Recuperado de http://www.leyes.congreso. gob.pe/

Contreras, C. (2012). La economía pública en el Perú después del guano y el salitre. Crisis fiscal y élites económicas durante su primer siglo independiente. Lima: Banco Central de Reserva del Perú, Instituto de Estudios Peruanos.

Dancuart, E. (1905). Anales de la Hacienda Pública del Perú. Historia y legislación fiscal de la República (t. VII). Lima: Librería e Imprenta Gil. 
FernÁNDEZ, G. (1985). El fin del Estado. Revista Universitaria (2).

FukUYAMA F. (2004). La construcción del Estado. Hacia un nuevo orden mundial en el siglo xxI. Lima: Ediciones B, S. A.

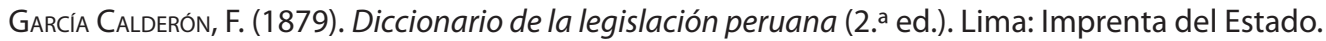

Idea Internacional y Transparencia. (2009). Gestión pública. Material de trabajo. Lima: Idea Internacional y transparencia.

LEÓN Y LEón, F. (1897). Tratado de derecho administrativo. (t. I). Lima: E. Moreno Editor.

LÓPEZ, S. (1997). Ciudadanos reales e imaginarios. Concepciones y mapas de la ciudadanía en el Perú. Lima: Instituto de Diálogo y Propuestas.

Manifestación del Estado de la Hacienda de la República del Perú en fin de abril de 1827. Presentada al Soberano Congreso Constituyente por el ciudadano encargado de la Dirección del Ministerio. (1827). Lima: Imprenta Rep. por J. M. Concha.

Manzanilla, J. M. (1903). El poder legislativo en el Perú. Anales Universitarios (31), 2-29.

McEvor, Carmen (1994). Un proyecto nacional en el siglo xix. Manuel Pardo y su visión del Perú. Lima: Pontificia Universidad Católica del Perú.

MiRó QuesAdA, O. (1912). La nacionalización de derecho por la extensión universitaria. Revista Universitaria, 7 (2).

Oviedo, J. (1861). Colección de leyes, decretos y órdenes publicadas en el Perú desde el año 1821 hasta el 31 de diciembre de 1859 (t. III). Lima: Felipe Bailly Editor.

PlACENCIA, L. (1985). Crecimiento del Ejecutivo durante el periodo de 1919-1930 (tesis para optar el grado académico de bachiller en Derecho). Pontificia Universidad Católica del Perú

Planas, P. (1993). El Estado moderno (Una nueva biografía). Lima: Desco.

Prado, J. (1984). Estado social del Perú durante la dominación española. Anales Universitarios (22), 1-159.

Prado, M. (1917). Proclamación de Maestro de la Juventud. Revista Universitaria (12).

QuintAnA, E. (1998). Empleos en propiedad en el Perú del siglo xix. Boletín del Instituto Riva-Agüero, (25) 325-363.

QuINONES, L. (2014). Construir y modernizar: el Ministerio de Fomento (1896-1930). Lima: Universidad Nacional de Ingeniería.

RAGAS, J. (2016). Ideólogos del Leviatán. Estadística y sociedad en el Perú (1791-1876). Estudios Sociales del Estado, 2(4), 14-34.

Rubıo, M. (1985). El Sistema Jurídico. Introducción al Derecho (2. a ed.). Lima: Pontificia Universidad Católica del Perú.

Saberbein, G. (1984). La planificación en el Perú. Antecedentes, desarrollo y técnicas. Lima: Editorial Imprenta Liberación S. A., Centro de Investigación Económica para la Acción.

TANTAlEÁn, J. (2004). Reforma del Estado y modernización de la gestión pública. En Ciudadanos por un Buen Gobierno (Ed.), Los nudos críticos de la gobernabilidad: Propuestas para un buen gobierno (pp. 171-207). Lima: Idea Internacional.

Trazegnies, F. De (1979). La idea de derecho en el Perú Republicano del siglo xix. Lima: Pontificia Universidad Católica del Perú.

\section{Bibliografía}

Aljovín de LosAdA, C. (2000). Caudillos y Constituciones. Perú: 1821-1845. Lima: Pontificia Universidad Católica del Perú, Instituto Riva Agüero, Fondo de Cultura Económica.

BéJAR, H. (2007). Justicia social, política social (3. a ed.). Lima: Cedep. 
Contreras, C. (2000). Centralismo y descentralismo en la historia del Perú independiente. Lima: JCAS, Instituto de Estudios Peruanos.

Del-Águila, A. (2012). La ciudadanía corporativa. Política, constituciones y sufragio en el Perú (1821-1896). Lima: Instituto de Estudios Peruanos.

Escalante, F. (2016). Historia mínima del neoliberlismo. Orígenes intelectuales de una revolución cultural. Lima: La Siniestra.

Gootenberg, P. (1995). Población y etnicidad en el Perú republicano (siglo xix). Algunas revisiones. Lima: Instituto de Estudios Peruanos.

Guzmán, C. (2002). Las relaciones de Gobierno entre el Poder Ejecutivo y el Parlamento. Lima: Pontificia Universidad Católica del Perú.

Peloso, V. y J. Ragas (2001). Estadística y sociedad en el Perú poscolonial: el desconocido censo de Lima de 1860. Histórica, 25(2), 275-293.

Rubio, M. (2009). El Sistema Jurídico. Introducción al Derecho (10. a ed. aumentada). Lima: Pontificia Universidad Católica del Perú.

TANTALEÁN, J. (1983). Política económica-financiera y la formación del Estado: siglo xix. Lima: Centro de Estudios para el Desarrollo y la Participación.

UGARTE, C. (1980 [1926]). Bosquejo de la historia económica del Perú. Lima: Banco Central de Reserva.

UGARTECHE, O. (2004). Adiós Estado, bienvenido mercado. Lima: Friedrich Ebert Stifung, Universidad Nacional Mayor de San Marcos. 